\title{
Quantized biopolymer translocation through nanopores: Departure from simple scaling
}

\section{Citation}

Melchionna, Simone, Massimo Bernaschi, Maria Fyta, Efthimios Kaxiras, and Sauro Succi. 2009. "Quantized Biopolymer Translocation through Nanopores: Departure from Simple Scaling."

Physical Review E 79 (3). https://doi.org/10.1103/physreve.79.030901.

\section{Permanent link}

http://nrs.harvard.edu/urn-3:HUL.InstRepos:41384107

\section{Terms of Use}

This article was downloaded from Harvard University's DASH repository, and is made available under the terms and conditions applicable to Open Access Policy Articles, as set forth at http:// nrs.harvard.edu/urn-3:HUL.InstRepos:dash.current.terms-of-use\#OAP

\section{Share Your Story}

The Harvard community has made this article openly available.

Please share how this access benefits you. Submit a story.

Accessibility 


\title{
Quantized biopolymer translocation through nanopores: departure from simple scaling
}

\author{
Simone Melchionna ${ }^{1,2}$, Massimo Bernaschi ${ }^{3}$, Maria Fyta ${ }^{1}{ }^{*}$, Efthimios Kaxiras ${ }^{1,4}$, and Sauro Succi ${ }^{4}$ \\ 1 Department of Physics and School of Engineering and Applied Sciences, \\ Harvard University, 17 Oxford Street, \\ Cambridge, MA 02138, USA \\ 2 INFM-SOFT, Department of Physics, \\ Università di Roma "La Sapienza", \\ P.le A. Moro 2, 00185 Rome, Italy \\ 3 Istituto Applicazioni Calcolo, CNR, \\ Viale del Policlinico 137, 00161, Roma, Italy \\ 4 Initiative in Innovative Computing, \\ Harvard University, Cambridge, MA, USA
}

(Dated: September 7, 2018)

\begin{abstract}
We discuss multiscale simulations of long biopolymer translocation through wide nanopores that can accommodate multiple polymer strands. The simulations provide clear evidence of folding quantization, namely, the translocation proceeds through multi-folded configurations characterized by a well-defined integer number of folds. As a consequence, the translocation time acquires a dependence on the average folding number, which results in a deviation from the single-exponent power-law characterizing single-file translocation through narrow pores. The mechanism of folding quantization allows polymers above a threshold length (approximately 1,000 persistence lengths for double-stranded DNA) to exhibit cooperative behavior and as a result to translocate noticeably faster.
\end{abstract}

The translocation of biopolymers through nanopores is drawing increasing attention because of its role in many fundamental biological processes, such as viral infection by phages, inter-bacterial DNA transduction or gene therapy [1]. This problem has motivated a number of in vitro experimental studies, aimed at exploring the translocation process through protein channels across cellular membranes [2, 3], or through micro-fabricated channels [4]. Recent experimental work has addressed the possibility of ultra-fast DNA-sequencing using electronic identification of DNA bases, while tracking its motion through nanopores under the effect of a localized electric field [5]. Experiments also reported that the translocation of biopolymers through pores wide enough to accommodate multiple strands, exhibits the intriguing phenomenon of current-blockade quantization, that is, discrete jumps of the electric current through the pore during the translocation process [6]. This was interpreted as indirect evidence that the polymer crosses the pore in the form of discrete configurations, associated with integer values of the folding number, that is, the number of strands simultaneously occupying the pore during the translocation. This behavior has been recently confirmed by direct observation of multi-folded configurations in large-scale simulations of biopolymer translocation [7].

In the present work, we report on the behavior of long polymers undergoing translocation through relatively wide pores, which exhibits qualitatively new features. Under these conditions, we predict from our simulations that folding quantization is actually enhanced and leads to faster translocation by effectively reducing friction through the pore region. The observed behavior also elicits an intriguing analogy with quantum systems, whereby the observed translocation time can be formulated as a weighted average over the whole set of multi-folded configurations (the "pure states" of the polymer-pore system). Within this picture, the translocation time acquires an additional dependence on the polymer length, through the average value of the folding number $\langle q\rangle_{N}$, which increases with the polymer length $N$. Thus, at variance with the case of narrow and short pores [8, 19, 10, 11, 12], translocation through wide pores allowing for multiple simultaneous strands is not described by a single power-law exponent. To give a specific example of scales involved, the size of the pores required to observe this behavior in double-stranded DNA is of the order of several $(\sim 10)$ times the effective cross-sectional diameter (which depends on salt concentration and repulsion between pairs of aligned DNA molecules), while the length threshold above which this behavior emerges is $\sim 150,000$ base pairs (bp's). What is remarkable and counter-intuitive about this behavior is, first, a highly ordered organization of the multiple strands at high folding number, and second, the ability of the quantized configurations to flow through the wide pore without experiencing any additional drag, compared to the single-file configuration.

Multiscale model: Our results are obtained from a multiscale treatment of translocation, involving a coarse-grained model for the biopolymer in which the basic unit ("bead") is equivalent to one persistence length ( $\sim 50 \mathrm{~nm})$, and the

\footnotetext{
* Present address: Department of Physics, Technical University of Munich, Germany
} 


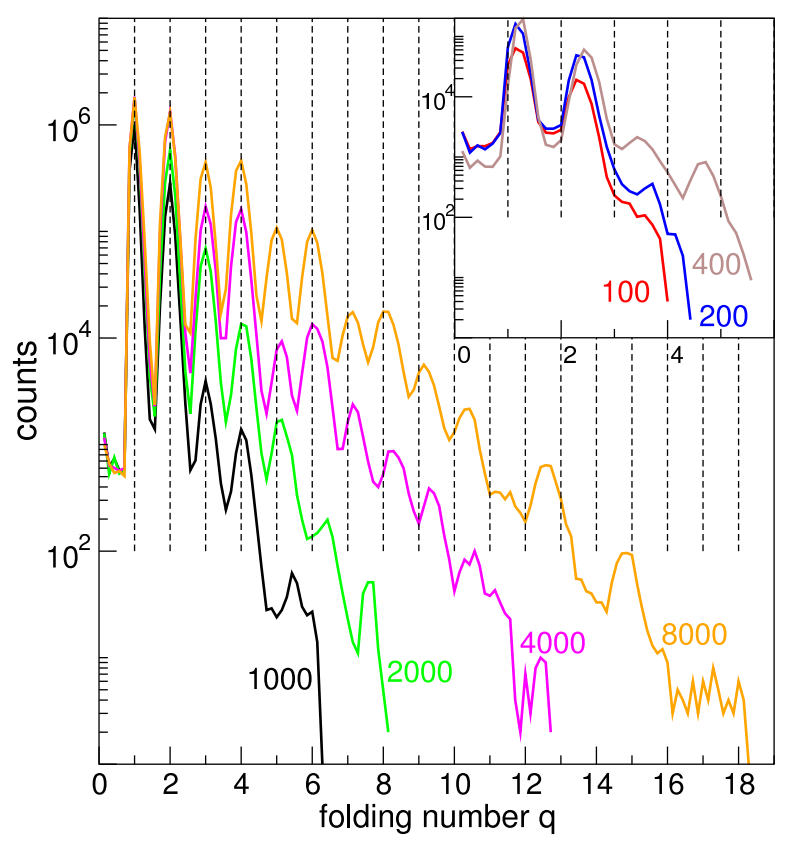

FIG. 1: (Color) Probability distribution of the folding number $q$ for polymer lengths $N=1,000-8,000$ and pore width $d_{p}=9$. Peaks at integer $q$ values (shown by vertical dashed lines) are evident. The inset shows data for $N=100-400$ at low- $q$ values.

molecular motion is coupled to the motion of the solvent in which the biopolymer exists. Incidentally, the length of polymers considered here, up to 8,000 beads (equivalent to $\sim 1.2 \times 10^{6} \mathrm{DNA}$ bp's), is an order of magnitude above any previous simulation in the field.

The model couples microscopic molecular dynamics (MD) for the biopolymer bead motion to a mesoscopic lattice Boltzmann (LB) [13] treatment of the solvent degrees of freedom [10]. In contrast to Brownian dynamics, the LB approach handles the fluid-mediated solvent-solvent interactions through an effective representation of local collisions between the solvent and solute molecules. The biopolymer translocates through a nanopore under the effect of a strong localized electric field applied across the pore ends, similar to the conditions in experimental settings [9], with the entire process taking place in the fast translocation regime.

A periodic box of size $N_{x} N_{y} N_{z}(\Delta x)^{3}$ lattice units, with $\Delta x$ the spacing between lattice points, contains both the solvent and the polymer. All parameters are measured in units of the LB time step and spacing, $\Delta t$ and $\Delta x$, respectively (both set to 1 ); the MD time step is 0.2 . We take $N_{x}=N_{y}=N_{z}$ with the wall at $x=\left(N_{x} / 2\right), N_{x}=128$ and the number of beads $N$ in the $100-8,000$ range. At $t=0$ the polymer resides on one side of the separating wall, $x>\left(N_{x} / 2\right)$, near the opening of a cylindrical pore of nominal length $l_{p}=3$ and nominal diameter $d_{p}$; we considered a narrower, $d_{p}=5$, pore and a wider one, $d_{p}=9$. Translocation is induced by a constant electric field acting along the $x$ direction and confined to a cylindrical channel of the same size as the pore, and length 3 along the streamwise $(x)$ direction. The pulling force associated with the electric field, $E$, in the experiments is $q_{e} E=0.02$ and the average thermal speed $k_{B} T / m=10^{-4}$, where $q_{e}$ is the effective charge per bead. The interaction between monomers and with the wall are modeled by 6-12 Lennard-Jones potentials [14], and other aspects of the simulation are the same as those in our previous work [10], which successfully reproduced single-file translocation [12, 15]. The effective width and radius of the surrounding pore must take into account the repulsive bead-wall interactions that result in an effective exclusion distance of $\simeq 1.5[\mathbf{1 0}]$. Therefore, a monomer is considered to lie inside the pore if contained in a pore of effective width $l^{e f f} \simeq 6$ (due to exclusion on both sides of the separating wall) and diameter $d^{e f f} \simeq 7.5$ for $d_{p}=9\left(d^{e f f} \simeq 3.5\right.$ for $\left.d_{p}=5\right)$. To measure the residence number of beads in the pore region we define a cylinder of length $h_{p}=10$ and radius $d_{p}$ centered at the pore midpoint and with axis aligned with the pore. This extended region misses monomers close to the pore openings and in contact with the wall, but permits to measure the number of beads in a wider region than the pore width with better statistics (reduced variation in the resident-bead number).

Configurational analysis - quantization of the folding number: In Fig 1. we show the cumulative statistics of the 


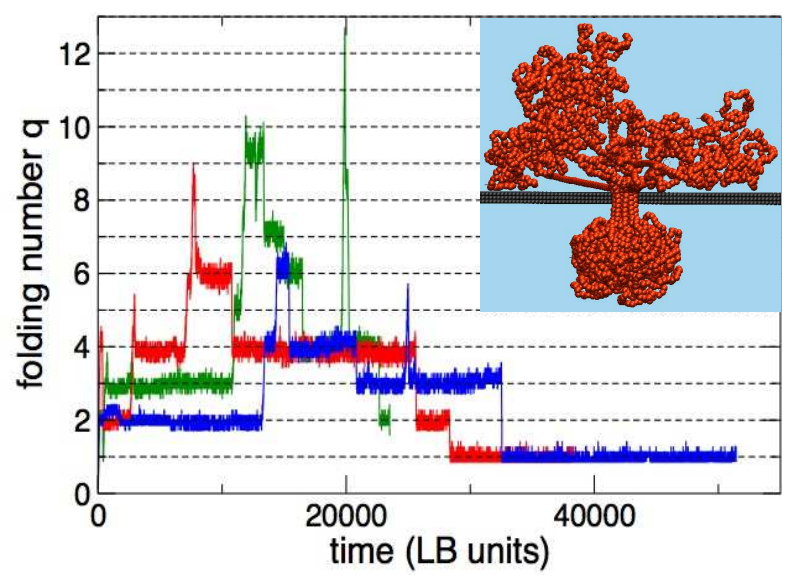

FIG. 2: (Color) Three trajectories of the folding number sampled from the ensemble $N=4,000$ and $d_{p}=9$. Low $q$ configurations are most often visited, but $q \geq 10$ can also occasionally occur. The inset shows an actual translocation event at the mid-point with $q=9$.

folding number, $q=N_{\text {res }} / N_{1}$, collected at each time-step of every single trajectory for a series of 100 realizations for each polymer length. Here, $N_{\text {res }}$ is the number of resident beads at each given time for each realization, while $N_{1}$ is the observed single-file value of the resident number, $N_{1}=h_{p} / b^{\text {eff }} \equiv h_{p}\left(1 / b+1 / \sigma_{b}\right) / 2 \sim 7$ (for values of $\sigma_{b}$ and $b$ see [14] and [15]). The combined statistics over initial conditions and time evolution, produces an aggregate ensemble, ranging from about $10^{5}$ time frames for the shortest history, $\left(N=100, d_{p}=9\right)$, up to over $10^{7}$ time frames for the longest one $\left(N=8,000, d_{p}=5\right)$. The case $d_{p}=9$ reveals a sharp quantization of the distribution of the folding number, with well defined peaks, which closely resembles the one observed in double-strand DNA translocation through solid nanopores (see Fig.7 of [16]). For the shortest strands, $N=100,200,400$, the peaks are shifted to values slightly larger than the integer values $q=1,2$. This indicates that the polymer spends most of its time between the low-fold states $q=1,2$. However, for longer strands $N>1,000$, the peaks of the distribution appear almost perfectly centered at integer values, up to $q=4,5,6$, respectively. This quantized spectrum is particularly evident for the case of the longest polymers, $N=4,000$ and 8,000 . In this case, "quantum states" up to $q=10$ are populated, with a slight shift of the peaks to values higher than integer values only for $q>7$. Note that the quantization is evident up to $q=10$, which is still significantly smaller than the largest folding number compatible with the pore diameter, $q_{\max }=\left(d_{p} / \sigma\right)^{2} \sim 20$ [17]. The value of $q$ above which the quantization gradually diverges from integer values, is an increasing function of the polymer length. The case $d_{p}=5$ (not presented) shows the same structure, though on a smaller range of $q$ values, up to $q=5$.

These data suggest an intriguing analogy with quantum systems, the folding number playing the role of the quantum numbers associated with excited states of atomic and molecular configurations. Within this analogy, single-file translocation $(q=1)$ would represent the analog of the ground state of the polymer-pore system. Indeed, the long-term, final stage of the translocation, is always found to proceed in single-file mode, corresponding to the polymer tail. In this respect, long polymers translocating through wide pores are naturally expected to exhibit a richer spectrum of excitations vs. short polymers translocating through narrow pores. In particular, the number of excited states supported by the polymer-pore system should grow quadratically with the pore diameter. Specifically, a polymer of length $L=b N$ translocating through a pore of length $l_{p}^{e f f}$ and diameter $d_{p}^{e f f}$, can produce a spectrum of folding numbers up to a maximum of $q_{\max } \propto\left(d_{p}^{e f f} / \sigma_{b}\right)^{2}$ strands (full-packing limit), each consisting of $N_{1}=l_{p}^{e f f} / b^{e f f}$ monomers. This limit can only be saturated by sufficiently long polymers, such that $N / N_{1} \gg q_{\max }$, namely $N \gg N_{p} \sim l_{p}^{\text {eff }}\left(d_{p}^{\text {eff }}\right)^{2} / b \sigma_{b}^{2}$, with $N_{p} \sim 100$ the saturation length in the present work.

The statistical picture presented above is supported by the dynamic trajectories. In Fig. 2, we show the timeevolution of the folding number $q(t)$ for three histories drawn at random from the pool of a hundred realizations of the $N=4,000$ system. A very rich dynamics, with sudden jumps between the various "excited states", is clearly visible. Interestingly, jumps occur both ways, from low to high $q$ and vice-versa, corresponding to absorption/emission of "fold-quanta". This indicates that translocation is not monotonic, but consists of a mixed sequence of folding and un-folding events. As anticipated, this sequence is always found to end up in single-file configuration, corresponding to the translocation of the polymer tail. Interestingly, the trajectories spend virtually all of their time in quantized states with very sharp transitions between them. A snapshot of such a quantized state for a highly folded configurations 


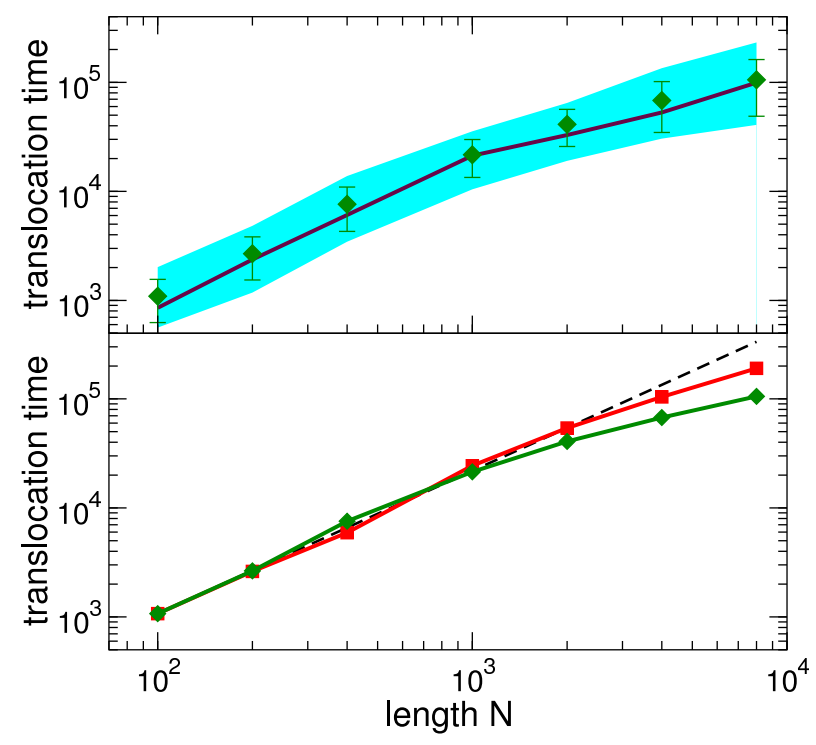

FIG. 3: (Color) Translocation times as a function of the polymer length $(N)$ for wide pore $\left(d_{p}=9\right)$. Top panel shows the average translocation time (green diamonds with associated error bars), the most probable translocation time (purple line) and the range between the maximum and minimum translocation times (shaded region). Bottom panel shows a linear fit to the low range results $10^{2}<N<10^{3}$ (dashed line), the multifile translocation time $\tau_{m f}(N)$ from Eq. (2) (red squares), and the average translocation time (green diamonds).

$(q=9)$ is shown in the inset of Fig. 2

Scaling exponents: In Fig. 3 we report the translocation time as a function of the polymer length, $N$, for translocation through the wide pore $\left(d_{p}=9\right)$. The most probable time from the time distributions, together with the average and the range between the minimum and maximum times are presented. From this figure, it is apparent that up to a length $N=1,000$, the translocation time $\tau$ obeys a scaling law of the form $\tau \sim N^{\alpha}$, with $\alpha \sim 1.36$ for the most-probable translocation time, slightly larger than the corresponding value for narrow pores [8, 9, 10, 11, 12]. We emphasize that, regardless of the exact values of the scaling exponents, all translocation indicators, that is, the minimum, maximum, most probable, and average translocation times, point clearly towards a deviation from a singleexponent power-law in the region $N>1,000$, a clear signature of multi-fold translocation. By restricting the analysis to the four longest chains, $N=1,000,2,000,4,000,8,000$, the bending of the curve (reduced translocation time) might be interpreted as the emergence of a new scaling exponent, $\alpha_{2} \sim 0.75$. However, as shown below, this bending is due to the multi-fold conformation of the translocating biopolymer, which does not necessarily follow a power-law dependence on the polymer size.

The translocation dynamics depends on the strength of the frictional forces exerted by the wall. In the single-file scenario, strong friction can change the power-law exponent from $\simeq 1.2$ to a linear relation $\tau \propto N$ [18]. In the case of multi-file translocation, a central issue is whether or not the highly folded configurations induce high-friction conditions. As demonstrated in Fig. 目 $d N / d t$ is linearly correlated to $q$, with approximately the same slope for all folds. If friction were dominant, $d N / d t$ vs. $q$ would asymptotically reach a constant value with increasing $q$, which is clearly not observed in the simulations. Moreover, the $d N / d t$ vs. $q$ slope depends only slightly on the polymer length (data not shown), changing by $\sim 30 \%$ in going from $N=400$ to $N=8,000$, which is further evidence in support of hydrodynamic coherence inside the pore. It is likely that such coherence arises from small velocity differences between neighboring beads in the pore, by minimizing bead-bead frictional forces, and/or the lubricating effect of the surrounding solvent enhanced by the alignment of strands. Therefore, frictional forces have a negligible effect, possibly limited to a small layer close to the wall, and unimportant for the group of translocating monomers. This rules out the possibility that the change of exponent is caused by frictional forces inside the pore.

Regardless of the underlying nature of the translocation process, we can compute the translocation time of each 


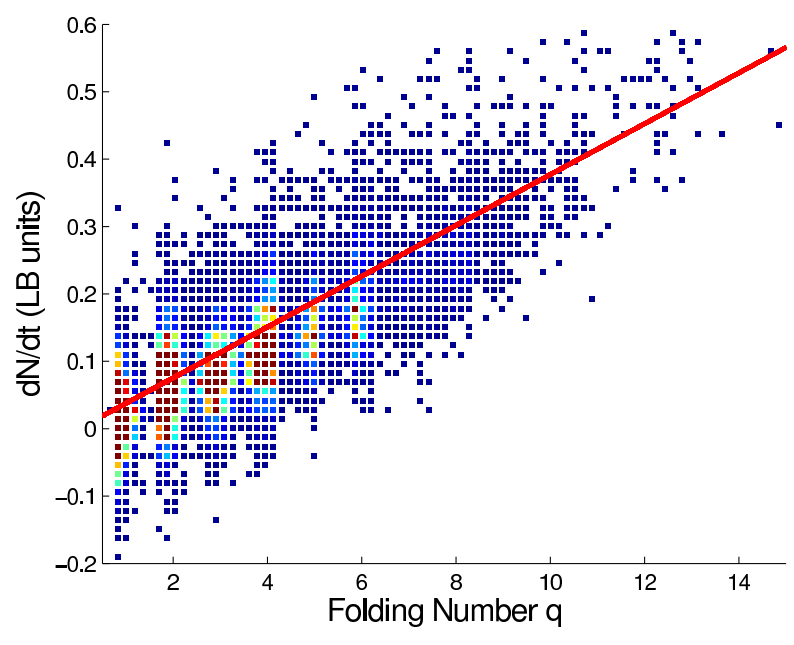

FIG. 4: (Color) Scatter plot illustrating the correlation between the folding number $q$ and the rate of translocating monomers $d N / d t$ for the wide pore $\left(d_{p}=9\right)$ and long polymer $(N=8,000)$ case. Colors indicate the density of points (blue: low, red: high); the straight line is the best linear fit.

realization, with $\tau$ the total translocation time:

$$
N=\int_{0}^{\tau} \frac{d N}{d t} d t=K_{N} \int_{0}^{\tau} N_{r e s}(t) d t
$$

where we have used the fact that $d N / d t$ and $N_{\text {res }}$ are linearly correlated with a constant of proportionality $K_{N}$. Next, we write $N_{\text {res }}(t)=q(t) N_{\text {res }, 1}$, where the subscript 1 stands for the single-file $(q=1)$ limit of the residence number. Eq. (11) then leads to $N=K_{N} N_{r e s, 1} \bar{q}_{N} \tau$, where $\bar{q}_{N}$ is the time averaged value of $q$. By averaging over all realizations, we obtain:

$$
\tau_{m f}(N) \sim \frac{N}{N_{r e s, 1}\left\langle K_{N}\right\rangle\left\langle\bar{q}_{N}\right\rangle} \equiv \frac{\tau_{1}(N)}{\left\langle\bar{q}_{N}\right\rangle}
$$

where brackets stand for ensemble averaging, $\tau_{m f}(N)$ is the multi-file translocation time, and we have defined $\tau_{1}(N) \equiv$ $\left(N / N_{\text {res }, 1}\right)\left(1 /\left\langle K_{N}\right\rangle\right)$; note that the dependence of $\left\langle K_{N}\right\rangle$ on $N$ is responsible for the non-linearity of $\tau_{1}(N)$. The simulation data show that the average $\left\langle\bar{q}_{N}\right\rangle$ remains approximately constant $\sim 1.2$, for $N<1,000$, and then begins growing, reaching $\sim 2.6$ for $N=8,000$. Therefore, we conclude that the departure of the translocation time from a power-law at large $N$ is mainly due to the increase of $\left\langle\bar{q}_{N}\right\rangle$ with polymer length, for $N>1,000$. This, in turn, results from the shift of the probability distribution of the translocation time towards higher $q$ values as $N$ is increased. For all lengths $N$ considered here, the time average $\left\langle\bar{q}_{N}\right\rangle$ remains below 3 , because the states $q=1$ and $q=2$ continue to be the most populated ones, for both pore diameters $d_{p}=5,9$. We have also checked that the high- $q$ peaks have a sizeable effect only on moments $\left\langle q^{p}\right\rangle$ for $p \geq 5$. Since the average translocation time is only a first order moment, the quantized peaks have little effect on it. This explains why the two pores, $d_{p}=5,9$, show similar dependence of $\left\langle\bar{q}_{N}\right\rangle$ on $N$. As a self-consistency check, in Fig. 3 we show the average translocation time for the case $d_{p}=9$, and compare it to the single-exponent estimate $\tau_{1}(N) \sim N^{1.31}$ and the compensated multi-file translocation time, $\tau_{m f}=\tau_{1}(N) /\langle\bar{q}\rangle_{N}$. The reasonable match of $\tau_{m f}$ with the data supports the idea that the speed-up of the longest chains can be attributed to the spectral shift of the folding number $q$.

Finally, we note that analyzing the in-pore conformation vs. $q$ is an important aspect of multi-file translocation. In the present communication we focused on how translocation, and the accompanying single-file out-of-pore hydrodynamics, is modulated by the population of the in-pore states, but have not analyzed in detail the in-pore conformations, which will be addressed in future work.

SM and MB acknowledge support by Harvard's Initiative in Innovative Computing. Computational resources were provided through the CyberInfrastructure Lab of the Harvard School of Engineering and Applied Sciences. We thank G. Lakatos for helpful comments. 
[1] H. Lodish, D. Baltimore, A. Berk, S. Zipursky, P. Matsudaira, and J. Darnell, Molecular Cell Biology (W.H. Freeman \& Co, NY, 1996).

[2] J.J. Kasianowicz et al., Proc. Nat. Acad. Sci. (USA), 93, 13770 (1996).

[3] A. Meller et al., Proc. Nat. Acad. Sci. (USA) 97, 1079 (2000).

[4] C. Dekker, Nature Nanotech., 2, 209, 2007.

[5] For a recent review of the field see D. Branton et al., Nature Nanotech., 26, 1, 2008.

[6] J. Li et al., Nat. Mater. 2, 611 (2003).

[7] M. Bernaschi, S. Melchionna, S. Succi, M Fyta, and E. Kaxiras, Nano Lett. 8, 1115 (2008).

[8] D.K. Lubensky and D.R. Nelson, Biophys. J. 77, 1824 (1999).

[9] A.J. Storm et al., Nano Lett. 5, 1734 (2005).

[10] M.G. Fyta, S. Melchionna, E. Kaxiras, and S. Succi, Multiscale Model. Simul. 5, 1156 (2006).

[11] C. Forrey and M. Muthukumar, J. Chem. Phys. 127, 015102 (2007).

[12] M. Fyta, S. Melchionna, S. Succi, E. Kaxiras, Phys. Rev. E 78, 036704 (2008).

[13] R. Benzi, S. Succi, and M. Vergassola, Phys. Rep. 222, 145, (1992).

[14] The Lennard-Jones parameters for bead-bead interactions are $\sigma_{b}=1.8, \epsilon_{b}=10^{-4}$ and cut-off distance 2.02 ; for bead-wall interactions $\sigma_{w}=1.5, \epsilon_{w}=10^{-3}$ and cut-off distance 1.68 .

[15] Briefly, the bonds between adjacent beads are modelled by springs with constant $k=0.5$ and equilibrium length $b=1.2$; the solvent density is $\rho=1$, kinematic viscosity $\nu=0.1$ and friction coefficient $\gamma=0.1$ (all in LB units). With $\Delta x=42$ $\mathrm{nm}, b$ becomes equal to the persistence length of double-stranded DNA $(50 \mathrm{~nm})$.

[16] A.J. Storm et al., Phys. Rev. E 71, 051903 (2005).

[17] To be precise, $q_{\max }=\left(d_{p}-\sigma_{w}\right)^{2} / \sigma_{b}^{2}=17.36$ for $d_{p}=9$; but beads can get closer to each other than $\sigma_{b}$ and closer to the wall than $\sigma_{w}$, giving $q_{\max } \sim 20$

[18] M. Zwolak and M. Di Ventra, Rev. Mod. Phys. 80, 141 (2008). 